\title{
Bifunctional Recombinant Protein Agent Based on Pseudomonas Exotoxin A Fragment for Targeted Therapy of HER2-Positive Tumors
}

\author{
S.M. Deyev, O.M. Kutova, E.N. Lebedenko, G.M. Proshkina, \\ A.A. Schulga and E.A. Sokolova
}

\begin{abstract}
Four variants of bifunctional HER2-specific recombinant proteins (anti-HER2-toxins) were created composed of HER2-specific scFv antibody (4D5scFv) or HER2-specific DARPin (D29) as targeting module and Pseudomonas exotoxin A fragment (PE40) as toxic module, and distinguished by the way of bacterial expression (cytoplasmic or periplasmic). Physicochemical, immunochemical, and functional properties of the created recombinant proteins as well as their expression yields were analyzed and compared with the option of the most promising one, D29-PE40, for further implementation as an agent for targeted therapy of HER2-overexpressing tumors.
\end{abstract}

Keywords Recombinant protein - HER2 receptor - Targeted therapy

Pseudomonas exotoxin A

\begin{abstract}
S.M. Deyev · O.M. Kutova · E.A. Sokolova $(\square)$
Institute of Biology and Biomedicine, Lobachevsky University,

Nizhny Novgorod, Russia

e-mail: malehanova@mail.ru

S.M. Deyev

e-mail: deyev@mail.ibch.ru

O.M. Kutova

e-mail: papicat@ rambler.ru

S.M. Deyev - E.N. Lebedenko - G.M. Proshkina - A.A. Schulga

Laboratory of Molecular Immunology, M.M. Shemyakin and Yu.A. Ovchinnikov Institute of Bioorganic Chemistry of the Russian Academy of Sciences (IBCh RAS), Moscow, Russia e-mail: elebedenko@mail.ru
\end{abstract}

G.M. Proshkina

e-mail: galina.proshkina@gmail.com
A.A. Schulga
e-mail: schulga@gmail.com
(C) The Author(s) 2018

K.V. Anisimov et al. (eds.), Proceedings of the Scientific-Practical Conference

“Research and Development - 2016”, https://doi.org/10.1007/978-3-319-62870-7_59 


\section{Introduction}

Development of new approaches and methods for cancer treatment presents one of the most important and rapidly developing areas of biology and medicine. The leading trend in this area is a personalized approach based on determining the molecular and genetic characteristics of the tumor in each particular case, and choosing the optimal method of treatment according to these characteristics. Due to progress in the study of the molecular basis of carcinogenesis, the targeted (or directed) therapy has been playing a greater role in recent decades, along with such main methods of cancer treatment as surgery and traditional chemotherapy. Targeted therapy implicates the use of bifunctional agents capable of selective binding to tumor cells expressing a specific molecular target (targeting function) and their elimination (therapeutic function). The tumor-selective action of such agents is intended to increase the therapeutic index values [9].

Membrane receptor HER2 (Human Epidermal growth factor Receptor 2) plays an important role in the process of epithelial cells malignant transformation, therefore being clinically relevant tumor marker. HER2-positive tumors are characterized by rapid growth and resistance to chemotherapy associating with a poor prognosis for the patient. HER2 overexpression typical for a number of carcinomas makes it also an advanced molecular target for targeted therapy that has been successfully proven [22].

Historically, the basis of targeted therapy is the use of monoclonal antibodies (MAbs). Their high affinity and specificity to target as well as antitumor action made it possible to create MAb-based drugs that have successfully passed clinical trials for the treatment of some tumors [9, 22]. There are two HER2-specific humanized MAbs used for targeted therapy of HER2-positive breast cancer, Trastuzumab (Herceptin) [7] and Pertuzumab (Omnitarg) [12], which are specific to different epitopes of the HER2 receptor. However, MAbs often fail to achieve optimum antitumor effect being used as monotherapy. Thus, prolonged use of Trastuzumab causes resistance to treatment in many patients [18], resulting in modifying the therapy strategy. Also, although a synergistic action of Trastuzumab and Pertuzumab, the latter is not effective against breast cancer cells resistant to Trastuzumab [19]. This problem has been addressed via conjugation of MAbs with other effector molecules: radioisotopes, low molecular and protein toxins, cytokines, etc., [20, 21]. In such immunoconjugates, antibody acts as a targeting component that delivers a cytotoxic one to target cells. Nevertheless, only one such HER2-specific immunoconjugate has been approved for the treatment of breast cancer, Trastuzumab emtansine (Kadsila), that is a conjugate of Trastuzumab and mitosis-inhibiting toxin of maytansinoid group [15]. The main difficulty of developing such immunoconjugates is chemical conjugation of targeting and toxic components (modules) that is characterized by a number of technical and scientific problems: lack of reproducibility and variability of the composition of the conjugates, possible decrease in the affinity of the targeting module or the effectiveness of the toxin, as well as admixture of nonconjugated molecules [11]. Solution to this 
problem is the usage of genetic engineering approach that enables to receive addressed bifunctional agents as recombinant proteins, i.e., fusion proteins comprising targeting and toxic modules [4, 23].

The targeting module of recombinant bifunctional agent may be presented by truncated antibody derivatives ( $\mathrm{scFv}$ and $\mathrm{dsFv}$ antibodies, nanobodies, ect.), or non-immunoglobulin scaffold proteins, such as DARPins (Designed Ankyrin Repeat Proteins). As toxic module, bacterial or plant protein toxins are of interest, particularly Pseudomonas exotoxin A which is one of the most potent protein toxins known to date [14]. The mechanism of Pseudomonas exotoxin A action consists in catalytic ADP-ribosylation of eukaryotic elongation factor 2 with subsequent inhibition of protein translation. Multidomain structure of Pseudomonas exotoxin A and functional independence of its domains facilitate the genetic engineering when construction of recombinant bifunctional agent.

We have created four variants of bifunctional HER2-specific recombinant proteins (anti-HER2-toxins) composed of HER2-specific scFv antibody (4D5scFv) or HER2-specific DARPin (D29) as targeting module and Pseudomonas exotoxin A fragment (PE40) as toxic module, and distinguished by the way of bacterial expression (cytoplasmic or periplasmic). Physicochemical, immunochemical, and functional properties of the created recombinant proteins as well as their expression yields were analyzed and compared with the option of the most promising one for further implementation as an agent for targeted therapy of HER2-overexpressing tumors.

\section{Research Description}

\section{Expression and Purification of Recombinant Proteins}

The following plasmids were used for bacterial production of recombinant proteins:

pET22-scFv4D5-PE40 plasmid with coding sequence of anti-HER2-toxin composed of Pseudomonas exotoxin A fragment (PE40) and HER2-specific antibody 4D5scFv (4D5scFv-PE40, or anti-HER2-toxin №1), and providing its cytoplasmic expression in E. coli cells, under control of inducible $T 7$ promoter;

pET22-D29-PE40 plasmid with coding sequence of anti-HER2-toxin composed of Pseudomonas exotoxin A fragment (PE40) and HER2-specific non-immunoglobulin scaffold protein DARPin D29 (D29-PE40, or anti-HER2-toxin №2), and providing its cytoplasmic expression in E. coli cells, under control of inducible $T 7$ promoter;

pSD-scFv4D5-PE40 plasmid with coding sequence of anti-HER2-toxin composed of Pseudomonas exotoxin A fragment (PE40) and HER2-specific antibody 4D5scFv (4D5scFv-PE40/p, or anti-HER2-toxin №3), and providing its periplasmic expression in E. coli cells, under control of inducible lac promoter;

pSD-D29-PE40 plasmid with coding sequence of anti-HER2-toxin composed of Pseudomonas exotoxin A fragment (PE40) and HER2-specific non-immunoglobulin 
scaffold protein DARPin D29 (D29-PE40/p, or anti-HER2-toxin №4), and providing its periplasmic expression in E. coli cells, under control of inducible lac promoter.

Both vectors, pET22 and pSD, contain the ampicillin resistance gene, which allows to select bacterial cells expressing the target protein. All expression cassettes contain sequence encoding oligohistidine tag at the C-terminus of the protein to facilitate subsequent protein purification by immobilized metal affinity chromatography using $\mathrm{Ni}^{2+}$-NTA-Sepharose. The pSD vector also contains ompA signal sequence, ensuring secretion of the target recombinant protein into the periplasmic space in order to both reduce its toxic effect on the cell and enhance the soluble fraction of the target protein.

Conditions used for expression of recombinant proteins №1-4 (strain, optical density of bacterial suspension at $550 \mathrm{~nm}$ before the induction $\left(\mathrm{A}_{550}\right)$, concentration of the inductor (isopropyl $\beta-\mathrm{D}$-1-thiogalactopyranoside, IPTG), induction time and temperature) are presented in Table 1.

After protein expression, bacterial cells were lysed by ultrasound and centrifuged. Then the target proteins were purified from the cleared lysate in two steps by $\mathrm{Ni}^{2+}$-chelate affinity chromatography using a $1 \mathrm{~mL}$ HisTrap FF column (GE Healthcare, USA) and ion exchange chromatography using a $1 \mathrm{~mL}$ Q Sepharose FF column (GE Healthcare, USA). Presence of the target proteins in elution fractions was controlled by denaturing electrophoresis in $12.5 \%$ polyacrylamide gel.

\section{Examination of physicochemical and immunochemical properties of the recombinant proteins}

The following procedures were carried out to test the structure of the proteins:

(1) verification of protein molecular weight by denaturing electrophoresis;

(2) checking the gene structure by sequencing;

(3) checking for presence of the address module $4 \mathrm{D} 5 \mathrm{scFv}$ or D29 by immunoblotting using antibodies specific to $4 \mathrm{D} 5 \mathrm{scFv}$ antibody or to DARPin D29, respectively;

(4) checking for presence of the toxic module by immunoblotting using antibodies specific to oligohistidine tag located at the C-terminus of the toxic module.

Table 1 Conditions of bacterial expression of four variants of anti-HER2-toxin

\begin{tabular}{l|l|l|l|l}
\hline \multirow{2}{*}{ Anti-HER2 toxin } & №1 & №2 & №3 & №4 \\
\cline { 2 - 5 } & 4D5scFv-PE40 & D29-PE40 & 4D5scFv-PE40/p & D29- PE40/p \\
\hline E. coli strain & C41 & BL21 (DE3) & SB536 & BL21 \\
\hline A 550 & 0.6 & 0.4 & 0.5 & 0.6 \\
\hline IPTG $(\mathrm{mM})$ & 1 & 1 & 0.5 & 1 \\
\hline Time $(\mathrm{h})$ & 4 & 4 & $14-16$ & 4 \\
\hline Temperature $\left({ }^{\circ} \mathrm{C}\right)$ & 28 & 37 & 28 & 37 \\
\hline
\end{tabular}


The $A_{280} / A_{260}$ ratio was determined spectrophotometrically. Checking the homogeneity of the proteins and their stability after 3 months storage was conducted by denaturing gel electrophoresis. Checking the content of bacterial endotoxins in the protein samples was conducted using limulous amoebocyte lysate test (LAL Test). Checking the specificity of interaction of the proteins with HER2 receptor was performed by ELISA using recombinant extracellular domain of the HER2 receptor as immobilized antigen.

\section{Study of Functional Properties of the Recombinant Proteins}

Equilibrium dissociation constant $\mathrm{Kd}$ of the recombinant proteins was determined on immobilized recombinant extracellular domain of the HER2 receptor by surface plasmon resonance using the BIAcore analyzer.

Cytotoxicity of the proteins was studied by the microculture tetrazolium test (MTT) [17] using adherent monolayer cell cultures expressing different amount of the HER2 receptor: SKBR-3 (HER2-overexpressing human breast adenocarcinoma, ATCC $^{\circledR}$ HTB-30 ${ }^{\mathrm{TM}}$ ) [13], SKOV-3 (HER2-overexpressing human ovarian adenocarcinoma, ATCC ${ }^{\circledR}$ HTB-77'M) [8], CHO (Chinese hamster ovary with no HER2 expression, $\mathrm{ATCC}^{\circledR}$ CCL-61 ${ }^{\mathrm{TM}}$ ) [16]. Data analysis and calculation of $\mathrm{IC}_{50}$ values were performed using the GraphPad Prism 6 software.

Antitumor effect of the recombinant protein D29-PE40 was estimated in vivo on intraperitoneal xenograft model of HER2-overexpressing human ovarian adenocarcinoma established in nude mice using ovarian cancer cells expressing far-red fluorescent protein TurboFP635. Monitoring of tumor growth was conducted by whole-body fluorescence imaging. To evaluate the efficacy of the anti-HER2-toxin, tumor growth inhibition coefficient (TGI) was calculated according to the following equation: $\mathrm{TGI} \%=\left[\left(\mathrm{Fl}_{\text {control }}-\mathrm{Fl}_{\text {experiment }}\right) \times 100 \%\right] / \mathrm{Fl}_{\text {control }}$, where $\mathrm{Fl}$ is an integral fluorescence intensity in the peritoneal cavity at a selected time point.

\section{Research Results}

We have created four bifunctional recombinant proteins (anti-HER2 toxins) as potential agents for targeted therapy of HER2-overexpressing tumors. These recombinant proteins are composed of targeting module specific to HER2 receptor and toxic module based on Pseudomonas exotoxin A. Targeting modules used are: (i) single chain antibody $4 \mathrm{D} 5 \mathrm{scFv}$ containing the variable domains of heavy and light chains of antibody Herceptin, which is widely used in clinical practice; (ii) innovative non-immunoglobulin scaffold polypeptide DARPin D29. DARPins present an alternative to antibodies as targeting molecules due to the following advantages: (i) smaller size of the protein, which facilitates the biochemical manipulation therewith; (ii) absence of cysteine residues in the structure and, as a 
result, aggregation stability and good expression; (iii) thermodynamic stability [5]. As a toxic module of the bifunctional recombinant proteins Pseudomonas exotoxin A fragment was used (PE40). This bacterial exotoxin is one of the most potent protein toxins known to date and has been successfully used for construction of a number of immunotoxins specific to different targets [2]. Two functional modules, targeting and toxic, were combined in a single recombinant protein by genetic engineering techniques. This approach to obtaining a bifunctional polypeptide has a number of advantages over commonly used chemical conjugation, primarily, precisely controlled composition, ease of production using bacterial producers and retention of functional properties of the modules. Bifunctional recombinant proteins were produced in bacteria that allows easy scaling of the technology for the laboratory and industrial production. In addition to the nature of the targeting module (4D5scFv or D29), the developed recombinant proteins vary in the way of expression in E. coli: cytoplasmic and periplasmic ones. The latter is aimed at increasing the level of recombinant protein expression in soluble form. Thus, four variants of anti-HER2-toxin were initially proposed (Table 2).

The recombinant proteins were expressed and purified. As was revealed by study of physicochemical and immunochemical properties of the produced proteins, each of them had expected molecular mass and structure, was highly pure, homogenous and storage stable as well as specific to HER2 receptor.

To quantify the affinity of the recombinants proteins for HER2 receptor, the equilibrium dissociation constant $\mathrm{Kd}$ was determined. All proteins were shown to have relatively equal $\mathrm{Kd}$ values lying in the low nanomolar range (Table 3) [24]. These particular Kd values are considered to be optimal when using targeted agents in vivo. Thus, very high-affinity scFv antibodies (with Kd values down to $10^{-11} \mathrm{M}$ ) demonstrate so-called «binding site barrier» effect that is stable binding of antibody to target receptors on tumor cells in the periphery of the tumor. As a result, such antibodies as well as targeted agents based on them poorly penetrate into the tumor tissue, so causing weak antitumor effect [1]. In the case of HER2-specific targeted

Table 2 Variants of anti-HER2 toxin

\begin{tabular}{|c|c|c|c|c|c|c|c|c|}
\hline № & Protein & L (a.a.) & $M(\mathrm{kDa})$ & Scheme of & gene construct & & & \\
\hline \multirow[t]{2}{*}{1} & \multirow[t]{2}{*}{ 4D5scFv-PE40 } & \multirow[t]{2}{*}{632} & \multirow[t]{2}{*}{68} & \multirow[t]{2}{*}{$\stackrel{\text { I7 }}{\longrightarrow}$} & & & & \\
\hline & & & & & 4D5scFv $\mathrm{H}$ & PE40 & $\mathrm{His}_{6} \mid \mathrm{K}$ & \\
\hline \multirow[t]{2}{*}{2} & \multirow[t]{2}{*}{ D29-PE40 } & \multirow[t]{2}{*}{540} & \multirow[t]{2}{*}{58} & \multirow[t]{2}{*}{$\stackrel{\mathrm{T} 7}{\longrightarrow}$} & & & & \\
\hline & & & & & D29 & PE 40 & $\mathrm{His}_{6} \mathrm{~K}$ & \\
\hline \multirow[t]{2}{*}{3} & \multirow[t]{2}{*}{ 4D5scFv-PE40/p } & \multirow[t]{2}{*}{642} & \multirow[t]{2}{*}{69.3} & \multicolumn{5}{|l|}{ lac $\mathrm{p} / \mathrm{o}$} \\
\hline & & & & & ompA $\quad \mathrm{His}_{6}$ & 4D5scFv & PE40 & $\mathrm{His}_{6} \mathbf{K}$ \\
\hline \multirow[t]{2}{*}{4} & \multirow[t]{2}{*}{ D29-PE40/p } & \multirow[t]{2}{*}{548} & \multirow[t]{2}{*}{59} & \multirow[t]{2}{*}{$\stackrel{\operatorname{lac} \mathrm{p} / \mathrm{o}}{\longrightarrow}$} & & & & \\
\hline & & & & & ompA $\mathrm{His}_{6}$ & D29 & PE 40 & Hiss $\mathrm{K}$ \\
\hline
\end{tabular}

$L$ number of amino acid residues in the protein; $M$ calculated molecular mass of the protein 
bifunctional agents loaded with a potent toxic module, too high affinity also leads to a significant increase in systemic toxicity: first, due to more efficient binding to normal cells with low expression of the target receptor, and second, because of the intrinsic shedding of the HER2 extracellular domain (i.e., its cleavage by extracellular metalloproteinases, or sheddases). HER2-specific agents of higher affinity bind more efficiently to the free HER2 extracellular domain in the bloodstream then being eliminated by reticuloendothelial cells and leading to high hepatic toxicity [6]. So, nanomolar affinity of all produced recombinant proteins for HER2 predetermines their optimal distribution and pharmacokinetics if used for HER2-specific targeted therapy.

High affinity shown for all recombinant proteins to HER2 receptor determined to a large extent their high cytotoxicity against HER2-expressing eukaryotic cells. The recombinant proteins were shown to potently inhibit growth of HER2-overexpressing cells SKOV-3 and SKBR-3 with $\mathrm{IC}_{50}$ values lying in the low picomolar range. At the same time, HER2-negative $\mathrm{CHO}$ cells were slightly affected with $\mathrm{IC}_{50}$ values lying in the range of nanomolar concentrations (Table 3) $[10,24]$.

Such a great difference in $\mathrm{IC}_{50}$ values for target (HER2-positive) and normal cells indicates a strongly pronounced selectivity of toxic effect of the proteins on HER2-expressing cells. This provides a basis for high therapeutic index values of the created proteins when using them as HER2-specific targeted agents in vivo.

Thus, all the created recombinant proteins demonstrated high potential for selective elimination of HER2-overexpressing cancer cells. We suppose that the nature of the HER2-specific targeting module actually did not affect functional activity of the bifunctional agent. However, the D29-PE40 recombinant protein was produced in $E$. coli most efficiently with the yield of its cytoplasmic expression of $160 \mathrm{mg}$ per liter of bacterial suspension that is several times higher than other protein variants (Table 3). Apparently, it is explained with DARP in structure that is free of cysteines. Cysteines residues are known to prevent a productive expression of the recombinant proteins in soluble and monomeric form due to (i) poor

Table 3 Comparative analysis of variants of anti-HER2 toxin

\begin{tabular}{|c|c|c|c|c|}
\hline \multirow[t]{4}{*}{ Anti-HER2 toxin } & \multicolumn{4}{|l|}{ Property } \\
\hline & 1 & 2 & 3 & \\
\hline & \multirow{2}{*}{$\begin{array}{l}\text { Protein yield, } \\
\text { mg per liter of } \\
\text { bacterial } \\
\text { culture }\end{array}$} & \multirow{2}{*}{$\begin{array}{l}\mathrm{Kd} \\
(\mathrm{nM})\end{array}$} & \multicolumn{2}{|l|}{$\mathrm{IC}_{50}(\mathrm{nM})$} \\
\hline & & & $\begin{array}{l}\text { HER2-overexpressing } \\
\text { cells }\end{array}$ & $\begin{array}{l}\text { HER2-negative } \\
\text { cells }\end{array}$ \\
\hline $\begin{array}{l}\text { №1 } \\
\text { (4D5scFv-PE40) }\end{array}$ & 25 & 5.6 & 0.0010 & 18 \\
\hline №2 (D29-PE40) & 160 & 11.3 & 0.0015 & 15 \\
\hline $\begin{array}{l}\text { №3 } \\
\text { (4D5scFv-PE40/p) }\end{array}$ & 15.2 & 5.6 & 0.0010 & 23 \\
\hline №4 (D29-PE40/p) & 37.5 & 11.3 & 0.0010 & 14 \\
\hline
\end{tabular}


formation of intramolecular disulfide bonds under the reducing conditions of the E. coli bacterial cytoplasm that interrupts proper protein folding and (ii) possible formation of intermolecular disulfide bonds leading to protein aggregation. Indeed, DARPins mostly have high level of cytoplasmic expression in E. coli in soluble form reaching up to $30 \%$ of total protein [25].

In view of the aforesaid, we considered the D29-PE40 recombinant protein as the optimal variant of anti-HER2 toxin and evaluated its antitumor efficacy in vivo on the HER2-overexpressing ovarian tumors established as intraperitoneal xenografts in athymic nude mice. To create this xenograft model, we used intraperitoneal human ovarian cancer cells expressing far-red fluorescent protein TurboFP635. Creation of fluorescent cancer cell lines presents an advanced approach in experimental oncology ensuring tumor growth visualization in living animal by fluorescence whole-body imaging [3]. Dynamics of intraperitoneal tumor growth was assessed by integral fluorescence intensity in the peritoneal cavity. The mice treated with $50 \mu \mathrm{g}$ D29-PE40 showed evident slowing down of tumor progression with the TGI value of $60 \%$ as compared to untreated (control) animals. The revealed antitumor effect against HER2-overexpressing tumor xenograft gives evidence of the therapeutic potential of the developed recombinant bifunctional protein D29-PE40 composed of HER2-specific DARPin and fragment of Pseudomonas exotoxin A.

\section{Conclusions}

The study resulted in creation of novel bifunctional HER2-specific recombinant protein (anti-HER2-toxin) based on HER2-specific DARPin and Pseudomonas exotoxin A fragment. The created anti-HER2 toxin was shown to selectively eliminate HER2-overexpressing cancer cells both in vitro and in vivo thus presenting a perspective agent for targeted therapy of HER2-positive tumors.

Acknowledgements Research was carried out with the financial support of the state represented by the Ministry of Education and Science of the Russian Federation. Agreement no. 14.578.21.0051, 23 Sent 2014. Unique project Identifier: RFMEFI57814X0051.

\section{References}

1. Adams, G.P., Schier, R., McCall, A.M., Simmons, H.H., Horak, E.M., Alpaugh, R.K., Marks, J.D., Weiner, L.M.: High affinity restricts the localization and tumor penetration of single-chain Fv antibody molecules. Cancer Res. 61, 4750-4755 (2001)

2. Alewine, C., Hassan, R., Pastan, I.: Advances in anticancer immunotoxin therapy. Oncologist 20(2), 176-185 (2015)

3. Balalaeva, I.V., Sokolova, E.A., Brilkina, A.A., Deyev, S.M., Petrov, R.V.: Far-red fluorescent cell line for preclinical study of HER2-targeted agents. Dokl. Biochem. Biophys. 465, 410-412 (2015) 
4. Becker, N., Benhar, I.: Antibody-based immunotoxins for the treatment of cancer. Antibodies 1(1), 39-69 (2012)

5. Binz, H.K., Amstutz, P., Kohl, A., Stumpp, M.T., Briand, C., Forrer, P., Grutter, M.G., Pluckthun, A.: High-affinity binders selected from designed ankyrin repeat protein libraries. Nat. Biotechnol. 22(5), 575-582 (2004)

6. Cao, Y., Marks, J.D., Huang, Q., Rudnick, S.I., Xiong, C., Hittelman, W.N., Wen, X., Marks, J.W., Cheung, L.H., Boland, K., Li, C., Adams, G.P., Rosenblum, M.G.: Single-chain antibody-based immunotoxins targeting Her2/neu: design optimization and impact of affinity on antitumor efficacy and off-target toxicity. Mol. Cancer Ther. 11(1), 143-153 (2012)

7. Carter, P., Presta, L., Gorman, C.M., Ridgway, J.B., Henner, D., Wong, W.L., Rowland, A. M., Kotts, C., Carver, M.E., Shepard, H.M.: Humanization of an anti-p185HER2 antibody for human cancer therapy. Proc. Natl. Acad. Sci. U S A 89(10), 4285-4289 (1992)

8. Dean, G.S., Pusztai, L., Xu, F.J., O’Briant, K., DeSombre, K., Conaway, M., Boyer, C.M., Mendelsohn, J., Bast Jr., R.C.: Cell surface density of p185(c-erbB-2) determines susceptibility to anti-p185(c-erbB-2)-ricin A chain (RTA) immunotoxin therapy alone and in combination with anti-p170(EGFR)-RTA in ovarian cancer cells. Clin. Cancer Res. 4(10), 2545-2550 (1998)

9. Deyev, S.M., Lebedenko, E.N., Petrovskaya, L.E., Dolgikh, D.A., Gabibov, A.G., Kirpichnikov, M.P.: Man-made antibodies and immunoconjugates with desired properties: function optimization using structural engineering. Russ. Chem. Rev. 84(1), 1-26 (2015)

10. Deyev, S.M., Lebedenko, E.N.: Supramolecular agents for theranostics. Bioorg Khim. 41(5), 539-552 (2015)

11. Ducry, L., Stump, B.: Antibody-drug conjugates: linking cytotoxic payloads to monoclonal antibodies. Bioconjug. Chem. 21, 5-13 (2010)

12. Franklin, M.C., Carey, K.D., Vajdos, F.F., Leahy, D.J., de Vos, A.M., Sliwkowski, M.X.: Insights into ErbB signaling from the structure of the ErbB2-pertuzumab complex. Cancer Cell 5(4), 317-328 (2004)

13. Hynes, N.E., Gerber, H.A., Saurer, S., Groner, B.: Overexpression of the c-erbB-2 protein in human breast tumor cell lines. J. Cell. Biochem. 39(2), 167-173 (1989)

14. Kreitman, R.J.: Immunotoxins for targeted cancer therapy. Aaps J. 8(3), 532-551 (2006)

15. Lambert, J.M., Chari, R.V.: Ado-trastuzumab Emtansine (T-DM1): an antibody-drug conjugate (ADC) for HER2-positive breast cancer. J. Med. Chem. 57(16), 6949-6964 (2014)

16. McCluskey, A.J., Olive, A.J., Starnbach, M.N., Collier, R.J.: Targeting HER2-positive cancer cells with receptor-redirected anthrax protective antigen. Mol. Oncol. 7(3), 440-451 (2013)

17. Mosmann, T.: Rapid colorimetric assay for cellular growth and survival: application to proliferation and cytotoxicity assays. J. Immunol. Methods 65, 55-63 (1983)

18. Nahta, R., Esteva, F.J.: Herceptin: mechanisms of action and resistance. Cancer Lett. 232, 123-138 (2006)

19. Nahta, R., Hung, M.C., Esteva, F.J.: The HER-2-targeting antibodies trastuzumab and pertuzumab synergistically inhibit the survival of breast cancer cells. Cancer Res. 64, $2343-$ 2346 (2004)

20. Panowski, S., Bhakta, S., Raab, H., Polakis, P., Junutula, J.R.: Site-specific antibody drug conjugates for cancer therapy. MAbs 6(1), 34-45 (2014)

21. Pastan, I., Hassan, R., FitzGerald, D.J., Kreitman, R.J.: Immunotoxin treatment of cancer. Annu. Rev. Med. 58, 221-237 (2007)

22. Polanovski, O.L., Lebedenko, E.N., Deyev, S.M.: ERBB oncogene proteins as targets for monoclonal antibodies. Biochemistry 77(3), 227-245 (2012)

23. Shapira, A., Benhar, I.: Toxin-based therapeutic approaches. Toxins 2(11), 2519-2583 (2010)

24. Sokolova, E.A., Stremovskiy, O.A., Zdobnova, T.A., Balalaeva, I.V., Deyev, S.M.: Recombinant immunotoxin 4D5scFv-PE40 for targeted therapy of HER2-positive tumors. Acta Naturae 7(4 (27)), 93-96 (2015)

25. Tamaskovic, R., Simon, M., Stefan, N., Schwill, M., Plückthun, A.: Designed ankyrin repeat proteins (DARPins): from research to therapy. Methods Enzymol. 503, 101-134 (2012) 
Open Access This chapter is licensed under the terms of the Creative Commons Attribution 4.0 International License (http://creativecommons.org/licenses/by/4.0/), which permits use, sharing, adaptation, distribution and reproduction in any medium or format, as long as you give appropriate credit to the original author(s) and the source, provide a link to the Creative Commons license and indicate if changes were made.

The images or other third party material in this chapter are included in the chapter's Creative Commons license, unless indicated otherwise in a credit line to the material. If material is not included in the chapter's Creative Commons license and your intended use is not permitted by statutory regulation or exceeds the permitted use, you will need to obtain permission directly from the copyright holder.

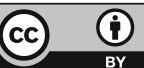

